\title{
Myocardial Infarction in an Adolescent Fontan Patient Who Consumed Energy Drinks on a Daily Basis and Stopped Thromboprophylaxis: A Case Report
}

\author{
Christoph Bauer ${ }^{1,2}$, Natascha Pramhofer ${ }^{1,2, ~ *, ~ M o u h a m a d ~ E l ~ H a j-M o u s t a f a ~}{ }^{1}$, Gerald Tulzer ${ }^{1,2}$ \\ ${ }^{1}$ Department of Paediatric Cardiology, Kepler University Hospital GmbH, Linz, Austria \\ ${ }^{2}$ Medical Faculty, Johannes Kepler University, Linz, Austria
}

Email address:

natascha.pramhofer@kepleruniklinikum.at (N. Pramhofer)

${ }^{*}$ Corresponding author

\section{To cite this article:}

Christoph Bauer, Natascha Pramhofer, Mouhamad El Haj-Moustafa, Gerald Tulzer. Myocardial Infarction in an Adolescent Fontan Patient Who Consumed Energy Drinks on a Daily Basis and Stopped Thromboprophylaxis: A Case Report. Cardiology and Cardiovascular Research. Vol. 5, No. 2, 2021, pp. 125-128. doi: 10.11648/j.ccr.20210502.20

Received: May 6, 2021; Accepted: May 26, 2021; Published: June 26, 2021

\begin{abstract}
Patients with single ventricle type congenital heart disease show a variety of risk factors for thromboembolic complications leading to stroke, pulmonary embolism and rarely myocardial ischemia. Energy drink consumption has been associated with adverse effects on vascular function and coagulation and may increase the risk. We report a case of a 14-year-old Fontan patient who presented with new onset cyanosis, chest pain and syncope. He quitted antiplatelet therapy himself three years ago and consumed three energy drinks a day regularly. On admission cardiac enzymes were severely elevated ECG showed slightly elevated ST-segments and Echo demonstrated reduced function. Myocarditis was first suspected but was rejected after MRI depicted a large transmural scar. During cardiac catheterization a total occlusion of the entire right coronary artery leading to myocardial infarction was found. The patient was put on dual anticoagulation and scheduled for transplantation because of severe myocardial impairment but did not consent and ultimately died. Myocardial infarction is an extremely rare complication in the Fontan population that has to be considered and ruled out in a timely manner in patients who present with chest pain and elevated troponin $\mathrm{T}$ to preserve ventricular function. Continuous thromboprophylaxis and avoidance of additional risk factors like energy drinks is warranted to prevent thromboembolic events in this population.
\end{abstract}

Keywords: Myocardial Infarction, Fontan Circulation, Energy Drink, Antiplatelet Therapy, Adolescent, Case Report

\section{Introduction}

Hypoplastic left heart syndrome is one of the most severe congenital heart defects. There is over 30 years of experience in treating this pathology [1]. In most cases this includes the three stage palliation with Norwood, Glenn and Fontan surgery [2]. Around 70.000 people are currently living with Fontan circulation around the world [1]. Overall, long-term survival shows a good prognosis. Nevertheless, their life expectancy remains shortened with significant cuts in the quality of life. This is caused by the elevated risk for thromboembolic events among other possible complications. Thromboembolic events after surgical palliation of single ventricle type congenital heart disease are rare but devastating complications. The stasis of blood flow caused by the Fontan circulation (i.e. the absence of a sub pulmonary pump) is the most important predisposing factor in these individuals [3]. Low cardiac output and alterations in the coagulation system may contribute to the increased lifelong risk of stroke and pulmonary embolism. Coronary artery affection leading to myocardial infarction (MI) is a very rare manifestation and has only been described in a few children, adolescent and young adults after the Fontan operation [4-6].

Energy drinks- a variable composition of vast amounts of caffeine, sugar, taurine, guarana, herbal extracts and other additions -have been associated with MI in other populations but not in Fontan patients. They have been shown to trigger vasospasm of e.g. coronary arteries increase platelet aggregation and decrease endothelial function and hence may promote thromboembolic complications in those already at 
risk [7-11].

We report a case of an adolescent Fontan patient who suffered a MI after consuming significant amounts of energy drinks and stopping thromboprophylaxis.

\section{Case Presentation}

An adolescent with a history of hypoplastic left heart syndrome who underwent a Norwood-, bidirectional Glennand an extracardiac fenestrated Fontan-operation at our facility presented at the age of 14 years with new onset cyanosis, chest pain and syncope. Until that event his medical course was unremarkable. The boy was physically fit and had no major medical conditions during the last few years. However, his last cardiac check-up was three years ago and he quitted antiplatelet therapy (acetylsalicylic acid, ASA) himself three years ago and consumed three energy drinks cans a day (each had $80 \mathrm{mg}$ of caffeine) for the last months. There was no current history of respiratory or gastrointestinal infections nor any family history of myocardial disease or thrombophilia.

Symptoms started in the morning while he was walking his dog. He suddenly felt a chest pressure, became dizzy and collapsed. His condition improved after a few minutes and hence he decided not to take the already sent ambulance to the hospital. During the next hours he was weak and tired. He felt increasingly short of breath and complained of pain in his chest and shoulders.

On arrival in our emergency department eight hours after onset of symptoms in the evening, the boy was tachypnoeic and tachycardic. Physical examination revealed normal heart and breath sounds, a blood pressure of $127 / 86 \mathrm{mmHg}$, and a normal capillary refilling time. His saturation was $94 \%$. ECG showed slightly increased ST elevation in precordial leads and negative $T$ waves in inferior leads (Figure 1). His echocardiography demonstrated a reduced left ventricular function and a reduced contractility of the lateral wall of the right ventricle. Cardiac enzymes (troponin T $(5189 \mathrm{ng} / \mathrm{L})$ ) and NT-proBNP (3295 ng/L) were significantly elevated.

Myocarditis was suspected and he was scheduled for cardiac MRI the next day. MRI (Figure 2) revealed a perfusion deficit and a large transmural late enhancement, which was not typical for myocarditis. Ejection fracture was reduced (EF $25.2 \%$ ) and the right ventricle dilated (end diastolic volume $179 \mathrm{ml}$, end systolic volume $133 \mathrm{ml}$ ). After considering the patients history and current test results decision was made to perform a cardiac catheterization to rule out MI.

During the investigation an almost total occlusion of the entire right coronary artery with minimal collateralization through the left coronary artery leading to MI of the anterolateral wall was found. (Figure 3) Percutaneous angioplasty was not an option since the onset of symptom had been more than 48 hours ago at that point. The patient was put on dual anticoagulation with phenprocoumon and ASA and received furosemide and lisinopril for his heart failure. Because of the vast myocardial involvement and the reduced myocardial function, the patient was advised to get listed for cardiac transplantation. During the next two weeks ventricular function improved slightly and the boy could be discharged. Three months later the patient had cardiac-arrest. Cardiopulmonary resuscitation was successfully initiated and he was admitted to the hospital. However, because of severe cardiac failure and refusal of cardiac transplantation he was ultimately sent to a palliative care unit where he died a view weeks later.

\section{Discussion}

Each patient with Fontan circulation shares a lifelong increased risk of thromboembolic events due to multiple risk factors [4]. In our patient thrombophilia was ruled out. There were no alterations of coagulation factors, no liver disease or signs of protein losing enteropathy but an area of potential blood stasis in the native aortic root. In addition, the patient had stopped the recommended antiplatelet therapy (ASA $100 \mathrm{mg} / \mathrm{d})$ by himself three years before. Although thromboprophylaxis is now recommended in most countries its significance and optimal therapy is not determined yet [12, 13]. Our institutional approach is to put all Fontan patients on ASA and those with presumed risk factors, previous thrombosis or older patients on anticoagulation. The lack of an effective thromboprophylactic therapy has contributed to the development of MI in this patient.

Another potential factor was his consumption of energy drinks. He consumed on average three energy drinks per day. Excessive intake of energy drinks have previously been reported to be associated with various cardiovascular impairments, including cardiac arrhythmias, prolonged QT interval and myocardial ischemia and infarction [14]. In the case of myocardial ischemia, evidence is still limited to a few case reports [6, 15-17]. A small prospective study demonstrated the detrimental effects of energy drinks on platelet and endothelial function most notable an acute increase of platelet aggregation and a decrease of endothelial function. This makes it reasonable to assume a causal relationship in our patient [14].

MI is an extremely rare complication in the Fontan population. Nevertheless, our case underlines the importance to consider and rule out this complication in a timely manner. An urgent cardiac catheterization is key to confirm diagnosis and to reopen blocked coronaries in order to preserve myocardium and cardiac function.

Coronary angioplasty was not an option anymore in our patient as cardiac catheterization was performed later than 48 hours of onset of symptom. This was due to the late admission of the patient to our hospital but also because his symptoms were initially interpreted as myocarditis which caused delay of cardiac catheterization. Retrospectively, we identified a few findings that could have earlier pointed towards a MI: the presenting symptoms with tightness in the chest, shortness of breath and syncope in the setting of an absent recent history of infection was not typical for myocarditis and the elevation of cardiac enzymes was higher than usually seen in myocarditis patients.

In our patient MI led to a continuous decrease in ventricular 
function. The option of cardiac transplantation was discussed with him and his parents. However, no consent could be reached, so he went on for palliative care and died several weeks later. Therefore, we emphasise the importance of considering MI in Fontan patients presenting with chest pain and syncope early on in the evaluation. To prevent thromboembolic events in this unique population an effective thromboprophylaxis and avoidance of additional potential risk factors like the intake of energy drinks may be important.

\section{Conclusion}

Energy drink consumption has been associated with cardiac arrythmias, increase of platelet aggregation, myocardial ischemia and infarction and should therefore be avoided in Fontan patients. Myocardial infarction is an extremely rare complication in Fontan patients and must be ruled out immediately in all patients who present with chest pain, ST segment abnormalities and significantly elevated troponin $\mathrm{T}$.

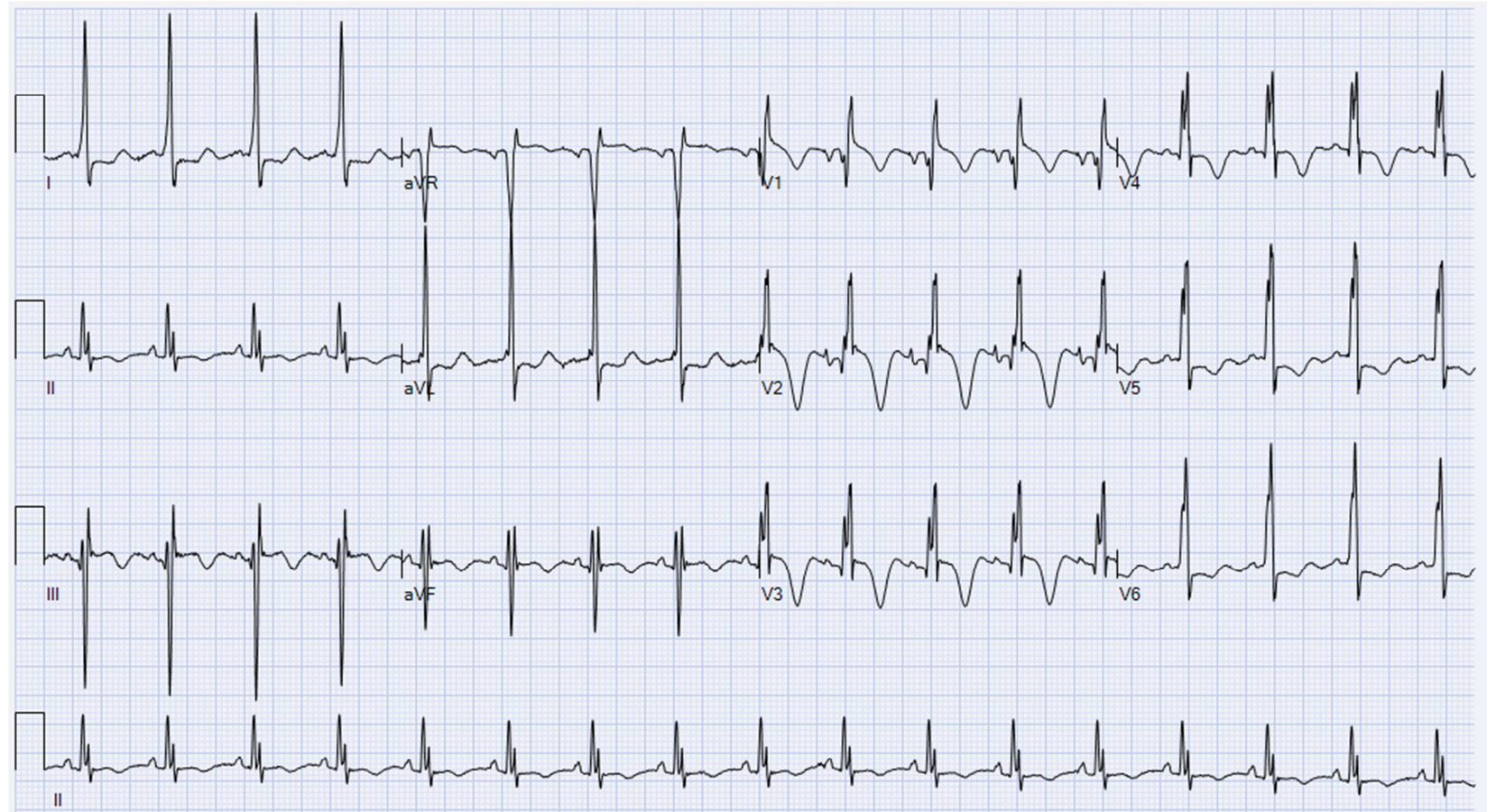

Figure 1. ECG of an adolescent Fontan patient with new onset chest pain showing elevated ST segments in V1-V3 and a negative T in II, III, aVF
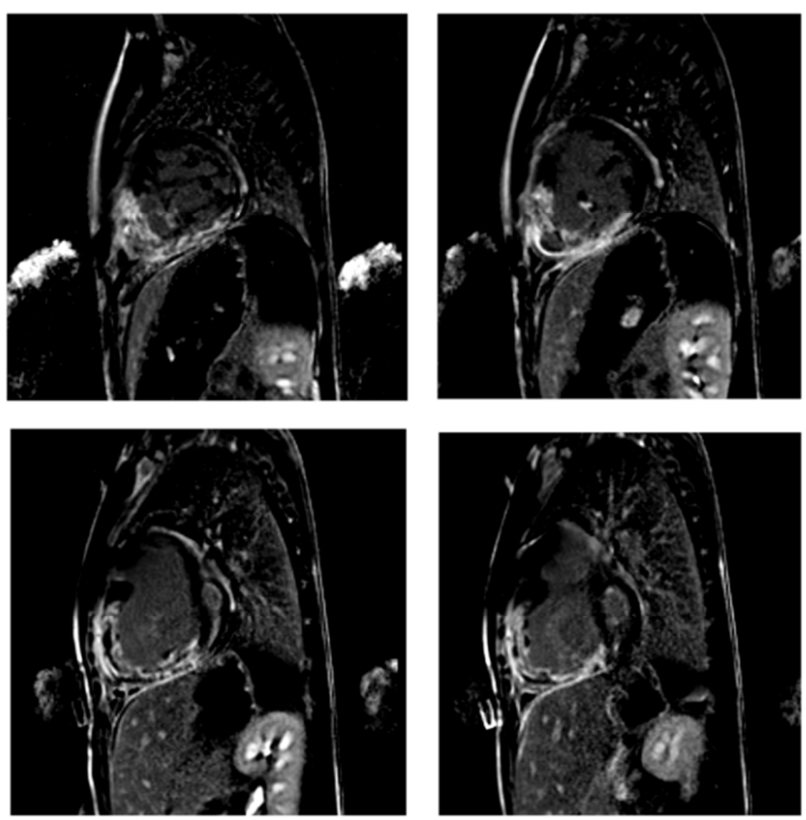

Figure 2. Cardiac MRI. Cardiac MRI (long axis section image series). Note transmural late enhancement apical and anterobasal

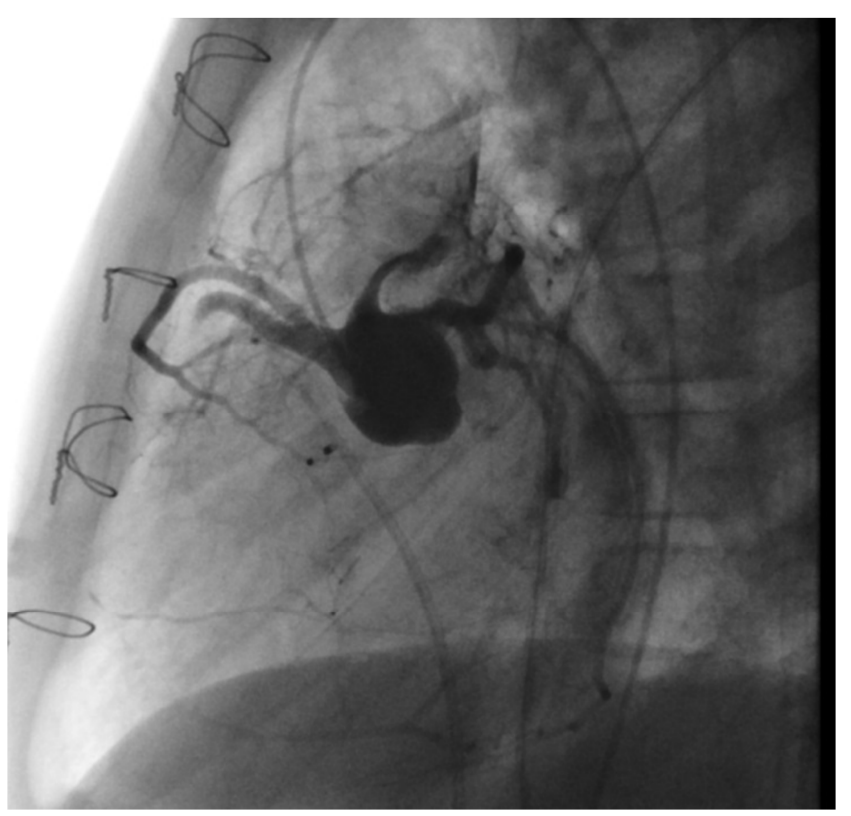

Figure 3. Cardiac catheterization, LAO projection showing total occlusion of $R C A$. 


\section{Ethical Standards}

For this case report no ethical clearance was obtained because it summarizes retrospective data that were exclusively collected for treatment and not for research.

\section{References}

[1] Metcalf MK, Rychik J. Outcomes in Hypoplastic Left Heart Syndrome. Pediatric Clinics of North America. 2020 Oct; 67 (5): 945-62.

[2] Roeleveld PP, Axelrod DM, Klugman D, Jones MB, Chanani NK, Rossano JW, et al. Hypoplastic left heart syndrome: from fetus to fontan. Cardiol Young. 2018 Nov; 28 (11): 1275-88.

[3] Chugh R. The Fontan Thromboprophylaxis Dilemma. Journal of the American College of Cardiology. 2019 Aug; 74 (8): 1082-5.

[4] Rychik J, Atz AM, Celermajer DS, Deal BJ, Gatzoulis MA, Gewillig MH, et al. Evaluation and Management of the Child and Adult with Fontan Circulation: A Scientific Statement From the American Heart Association. Circulation [Internet]. 2019 Aug 6 [cited 2021 May 6]; 140 (6). Available from: https://www.ahajournals.org/doi/10.1161/CIR.0000000000000 696.

[5] Deshaies C, Hamilton RM, Shohoudi A, Trottier H, Poirier N, Aboulhosn J, et al. Thromboembolic Risk After Atriopulmonary, Lateral Tunnel, and Extracardiac Conduit Fontan Surgery. Journal of the American College of Cardiology. 2019 Aug; 74 (8): 1071-81.

[6] Misra A, Gayanilo M, Gupta P. Coronary artery embolism in a patient with Fontan palliation: a rare complication. Cardiol Young. 2020 Jul; 30 (7): 1029-31.

[7] Somers KR, Svatikova A. Cardiovascular and Autonomic Responses to Energy Drinks-Clinical Implications. JCM. 2020 Feb 5; 9 (2): 431
[8] Pommerening MJ, Cardenas JC, Radwan ZA, Wade CE, Holcomb JB, Cotton BA. Hypercoagulability after energy drink consumption. Journal of Surgical Research. 2015 Dec; 199 (2): 635-40.

[9] Higgins JP, Babu K, Deuster PA, Shearer J. Energy Drinks: A Contemporary Issues Paper. Curr Sports Med Rep. 2018 Feb; 17 (2): 65-72.

[10] Lippi G, Cervellin G, Sanchis-Gomar F. Energy Drinks and Myocardial Ischemia: A Review of Case Reports. Cardiovasc Toxicol. 2016 Jul; 16 (3): 207-12.

[11] Mangi MA, Rehman H, Rafique M, Illovsky M. Energy Drinks and the Risk of Cardiovascular Disease: A Review of Current Literature. Cureus. 2017 Jun 7; 9 (6): e1322.

[12] Small AJ, Aboulhosn JA, Lluri G. Thromboprophylaxis in Adults with Atrio-Pulmonary Fontan. World J Pediatr Congenit Heart Surg. 2018; 9 (5): 504-8.

[13] Firdouse M, Agarwal A, Chan AK, Mondal T. Thrombosis and Thromboembolic Complications in Fontan Patients: A Literature Review. Clin Appl Thromb Hemost. 2014 Jul; 20 (5): 484-92.

[14] Worthley MI, Prabhu A, De Sciscio P, Schultz C, Sanders P, Willoughby SR. Detrimental effects of energy drink consumption on platelet and endothelial function. Am J Med. 2010 Feb; 123 (2): 184-7.

[15] Israelit SH, Strizevsky A, Raviv B. ST elevation myocardial infarction in a young patient after ingestion of caffeinated energy drink and ecstasy. World Journal of Emergency Medicine. 2012; 3 (4): 305.

[16] Hill D, Waldman A, Vivek D. A 16-year-old with ST elevation myocardial infarction: case report and review of the literature. Cardiol Young. 2016 Feb; 26 (2): 230-6.

[17] Subahi A, Forbes T, Ali OE. Percutaneous coronary intervention following Fontan procedure. IJC Heart \& Vasculature. 2020 Jun; 28: 100511. 